\title{
Doubly Non-Central Generalized Beta Distributions
}

\author{
DAYA K. NAGAR, EDWIN ZARRAZOLA AND YOHANA TABARES-HERRERA \\ Instituto de Matemáticas \\ Universidad de Antioquia \\ Calle 67, No. 53-108, Medellín \\ COLOMBIA
}

Abstract: - The doubly non-central generalized beta type 1 and type 2 distributions have been derived by using two independent non-central gamma variables with different scale parameters. These distributions generalize several well known central and non-central beta distributions. The doubly non-central generalized beta densities are much more flexible than many exiting beta models and can assume a large variety of shapes. Several properties of these distributions have been studied.

Key-Words: - Beta distribution; confluent hypergeometric function; gamma distribution; Humbert's confluent hypergeometric function; non-central distribution.

Received: October 11, 2020. Revised: February 16, 2021. Accepted: March 1, 2021. Published: March 19, 2021.

\section{Introduction}

The two-parameter univariate beta distribution is one of the most frequently used distributions and has been studied extensively in the scientific literature. Since the beta distribution features two shape parameters, there is high shape flexibility for various combinations of parameter values. For basic properties, the reader may consult Bury [1, Chapter 14], and Johnson Kotz and Balakrishnana[2, Chap. 24]. Also, Some relevant studies can be found in Kala [3] and Liu and Miao [4].

The beta distribution can be used to model the distribution of measurements whose values lie between zero and one. This distribution can also be used to represent proportion or probability outcomes. In Bayesian analysis, this distribution is taken as a conjugate prior in binomial sampling models. The well known uniform distribution is a special case of the beta distribution. The distribution of the order statistic from a uniform distribution on $(0,1)$ is beta. Further, several test statistics are functions of beta variables.

It is well know that if $X_{1}$ and $X_{2}$ are independent standard gamma random variables with respective shape parameters $a$ and $b$, then the random variable $U=X_{1} /\left(X_{1}+X_{2}\right)$ has a standard beta distribution (type 1$)$ with two parameters, denoted by $U \sim \mathrm{B} 1(a, b)$.

If independent random variables $X_{1}$ and $X_{2}$ have two parameter gamma distributions, $X_{1} \sim \mathrm{Ga}\left(a, \theta_{1}\right)$ and $X_{2} \sim \mathrm{Ga}\left(b, \theta_{2}\right)$, with densities

$$
\begin{aligned}
f_{X_{1}}\left(x_{1} ; a, \theta_{1}\right)= & \frac{\exp \left(-x_{1} / \theta_{1}\right) x_{1}^{a-1}}{\theta_{1}^{a} \Gamma(a)}, \\
& x_{1}>0, \quad a>0, \quad \theta_{1}>0
\end{aligned}
$$

and

$$
\begin{aligned}
f_{X_{2}}\left(x_{2} ; b, \theta_{2}\right)= & \frac{\exp \left(-x_{2} / \theta_{2}\right) x_{2}^{b-1}}{\theta_{2}^{b} \Gamma(b)}, \\
& x_{2}>0, \quad b>0, \quad \theta_{2}>0,
\end{aligned}
$$

then the density function of $U=X_{1} /\left(X_{1}+X_{2}\right)$ has the form

$$
\begin{gathered}
f_{\mathrm{GB} 1}(u ; a, b ; \lambda)=\frac{\lambda^{a} u^{a-1}(1-u)^{b-1}}{B(a, b)[1-(1-\lambda) u]^{a+b}}, \\
0<u<1,
\end{gathered}
$$

where $\lambda=\theta_{2} / \theta_{1}$ and $B(a, b)$ is the usual beta function.

The above distribution was first derived by Libby and Novic [5]. Chen and Novic [6] called this distribution a G3B distribution and studied several of its properties.

It is interesting to note that through the addition of a single scale-like parameter, a distribution is provided that can take on a wide range of values for the mean, variance, skewness, and kurtosis.

One can easily check that for $\lambda=1$ the density (3) reduces to a standard beta type 1 density and for $\lambda=2$ it slides to a beta type 3 density studied by Cardeño, Nagar and Sánchez [7], Gupta and Nagar [8], Nagar and Ramirez-Vanegas [9, 10], and Nagar and TabaresHerrera [11].

The density of the quotient $V=X_{1} / X_{2}$, where $X_{1}$ and $X_{2}$ are independent and follow two parameter gamma distributions defined by (11) and (2), can be obtained directly from the density of $U$. Since, we have $V=U /(1-U)$, we can obtain the density of $V$ from (3) as

$$
f_{\mathrm{GB} 2}(v ; a, b ; \lambda)=\frac{\lambda^{a} v^{a-1}}{B(a, b)(1+\lambda v)^{a+b}}, v>0 .
$$


It is easy to observe that for $a=\nu_{1} / 2, b=\nu_{2} / 2$ and $\lambda=\nu_{1} / \nu_{2}$ with $\nu_{1}>0, \nu_{2}>0$, the GB2 in (⿶) becomes a Snedecor $F\left(\nu_{1}, \nu_{2}\right)$ distribution while for $\lambda=1$, it is simply a standard type 2 beta distribution (inverted beta distribution). Also, for $a=1$, the GB2 distribution slides to a Lomax distribution. In general, $(\lambda b / a) V$ has a Snedecor $F(2 a, 2 b)$ distribution and the distribution of $\lambda V$ is standard beta type 2 .

The presence of the parameter $\lambda$ allows threeparameter generalized beta distributions to take a much wider variety of shapes than the standard beta distributions and therefore GB1 and GB2 distributions are more flexible than standard beta distributions and have been used in statistical modeling and applications. The first is used in several statistical models, e.g. in fitting utility functions (Libby and Novic [5]), in Bayesian analysis with binomial sampling (Chen and Novic [6]) etc., while the second is used in the analysis of carcinogenesis data, in the study of system availability or in measuring information in predictive distributions. Systematic studies of these distributions including problem of parameter estimation was done by Pham-Gia and Duong [12]. While defining the three-parameter generalized beta distributions, Libby and Novic [5] have also given their multivariate (bivariate) generalizations. The non-central counterpart of Libby and Novic's bivariate beta distribution is given in Gupta, OrozcoCastañeda and Nagar [13]. Sarabia and Castillo [14] have proposed several bivariate extensions of Libby and Novic's three-parameter generalized beta type 1 distribution. Also, for some pertinent and interesting generalizations of beta models, the reader is referred to Chen and Singh [15], Gómez-Déniz and Sarabia [16] and Alshkaki [17]. For a systematic treatment of matrix variate beta distribution the reader is referred to Gupta and Nagar [18].

Although in recent years, several generalizations of beta distribution have been developed to achieve more flexibility, not much work has been done in the area of non-central beta distributions (Johnson, Kotz and Balakrishnana [2], Nagar and RamirezVanegas [9, 10], Firmino et al. [19], Nadarajah [20]).

The non-central beta densities are usually obtained by using non-central gamma variables. The random variable $X$ is said to have a non-central gamma distribution with parameters $\kappa(>0), \theta(>0)$ and $\delta(\geq 0)$, denoted by $X \sim \mathrm{Ga}(\kappa, \theta, \delta)$, if its p.d.f. is given by

$$
\left\{\theta^{\kappa} \Gamma(\kappa)\right\}^{-1} \exp \left(-\delta-\frac{x}{\theta}\right) x^{\kappa-1}{ }_{0} F_{1}\left(\kappa ; \frac{\delta x}{\theta}\right),
$$

where $x>0$ and ${ }_{0} F_{1}(c ; z)$ is defined as

$$
{ }_{0} F_{1}(c ; z)=\sum_{i=0}^{\infty} \frac{\Gamma(c) z^{i}}{\Gamma(c+i) i !} .
$$

For $\delta=0$, the non-central gamma distribution reduces to a gamma distribution.

If independent random variables $X_{1}$ and $X_{2}$ have non-central gamma distributions, $X_{1} \sim \mathrm{Ga}\left(a, \theta_{1}, \delta_{1}\right)$ and $X_{2} \sim \mathrm{Ga}\left(b, \theta_{2}, \delta_{2}\right)$, then $U=X_{1} /\left(X_{1}+X_{2}\right)$ follows a doubly non-central generalized beta type 1 distribution. Likewise, the quotient $V=X_{1} / X_{2}$ follows a doubly non-central generalized beta type 2 distribution. For $\theta_{1}=\theta_{2}$ these distributions reduce to standard doubly no-central beta distributions.

In this article, we will derive and study doubly non-central generalized beta type 1 and 2 distributions. Since, the distributions defined in this article have increased number of parameters guaranteeing more flexibility than many existing beta models, they can serve as an alternative to many beta distributions and can be applied in various real-life problems.

\section{Some Definitions and Preliminary Results}

In this section, we give some definitions and preliminary results that have been used in subsequent sections. Throughout this work, we will use the Pochammer symbol $(a)_{n}$ defined by $(a)_{n}=a(a+1) \cdots(a+$ $n-1)=(a)_{n-1}(a+n-1)$ for $n=1,2, \ldots$, and $(a)_{0}=1$. The integral representations of the confluent hypergeometric function and the Gauss hypergeometric function are given as (Luke [21]]):

$$
\begin{aligned}
{ }_{1} F_{1}(a ; c ; z)= & \frac{\Gamma(c)}{\Gamma(a) \Gamma(c-a)} \\
& \times \int_{0}^{1} t^{a-1}(1-t)^{c-a-1} \exp (z t) \mathrm{d} t,
\end{aligned}
$$

and

$$
\begin{aligned}
{ }_{2} F_{1}(a, b ; c ; z)= & \frac{\Gamma(c)}{\Gamma(a) \Gamma(c-a)} \\
& \times \int_{0}^{1} \frac{t^{a-1}(1-t)^{c-a-1}}{(1-z t)^{b}} \mathrm{~d} t,
\end{aligned}
$$

respectively, where $\operatorname{Re}(a)>0$ and $\operatorname{Re}(c-a)>0$. Using power series expansion of $\exp (z t)$ in $(5)$ and $(1-z t)^{-a}$ in $(6)$, the series expansions of ${ }_{1} F_{1}(a ; c ; z)$ and ${ }_{2} F_{1}(a, b ; c ; z)$ are obtained as

$$
{ }_{1} F_{1}(a ; c ; z)=\sum_{k=0}^{\infty} \frac{(a)_{k}}{(c)_{k}} \frac{z^{k}}{k !}
$$

and

$$
{ }_{2} F_{1}(a, b ; c ; z)=\sum_{k=0}^{\infty} \frac{(a)_{k}(b)_{k}}{(c)_{k}} \frac{z^{k}}{k !}, \quad|x|<1 .
$$

The Humbert's confluent hypergeometric function 
$\Psi_{2}$ is defined by

$$
\begin{aligned}
\Psi_{2}\left[a ; c_{1}, c_{2} ; z_{1}, z_{2}\right] & =\sum_{r, s=0}^{\infty} \frac{(a)_{r+s}}{\left(c_{1}\right)_{r}\left(c_{2}\right)_{s}} \frac{z_{1}^{r} z_{2}^{s}}{r ! s !}, \\
& =\sum_{r=0}^{\infty} \frac{(a)_{r}}{\left(c_{1}\right)_{r}} \frac{z_{1}^{r}}{r !}{ }_{1} F_{1}\left(a+r ; c_{2} ; z_{2}\right) \\
& =\sum_{s=0}^{\infty} \frac{(a)_{s}}{\left(c_{2}\right)_{s}} \frac{z_{2}^{s}}{s !}{ }_{1} F_{1}\left(a+s ; c_{1} ; z_{1}\right),
\end{aligned}
$$

where $\left|z_{1}\right|<\infty$ and $\left|z_{2}\right|<\infty$. The integral representation of $\boldsymbol{\Phi}_{1}$ is given by

$$
\begin{aligned}
& \Psi_{2}\left[a ; c_{1}, c_{2} ; z_{1}, z_{2}\right] \\
& =\frac{1}{\Gamma(a)} \int_{0}^{\infty} \exp (-t) t^{a-1}{ }_{0} F_{1}\left(c_{1} ; t z_{1}\right)_{0} F_{1}\left(c_{2} ; t z_{2}\right) \mathrm{d} t \\
& \left|z_{1}\right|<\infty, \quad\left|z_{2}\right|<\infty
\end{aligned}
$$

where $\operatorname{Re}(a)>0$. For properties and further results on these functions the reader is referred to Luke [21] and Srivastava and Karlsson [22].

\section{Doubly Non-Central Generalized Beta Distributions}

In this section, doubly non-central generalized beta type 1 and 2 densities are derived and different shapes of these densities are shown graphically.

Theorem 3.1. Let $X_{1}$ and $X_{2}$ be independent random variables, $X_{1} \sim \mathrm{Ga}\left(a, \theta_{1}, \delta\right)$ and $X_{2} \sim \mathrm{Ga}\left(b, \theta_{2}, \mu\right)$. Then, $U=X_{1} /\left(X_{1}+X_{2}\right)$ follows a doubly noncentral generalized beta type 1 distribution with the $p d f$

$$
\begin{aligned}
& f_{\mathrm{NCGB} 1}(u ; a, b ; \lambda ; \delta, \mu) \\
& =\frac{\lambda^{a} \exp [-(\delta+\mu)]}{B(a, b)} \frac{u^{a-1}(1-u)^{b-1}}{[1-(1-\lambda) u]^{a+b}} \\
& \quad \times \Psi_{2}\left[a+b ; a, b ; \frac{\delta \lambda u}{1-(1-\lambda) u}, \frac{\mu(1-u)}{1-(1-\lambda) u}\right]
\end{aligned}
$$

where $0<u<1$ and $\lambda=\theta_{2} / \theta_{1}$.

Proof. Using independence, the joint pdf of $X_{1}$ and $X_{2}$ is given by

$$
\begin{gathered}
K \exp \left[-\left(\frac{x_{1}}{\theta_{1}}+\frac{x_{2}}{\theta_{2}}\right)\right] x_{1}^{a-1} x_{2}^{b-1} \\
\quad \times{ }_{0} F_{1}\left(a ; \frac{\delta x_{1}}{\theta_{1}}\right){ }_{0} F_{1}\left(b ; \frac{\mu x_{2}}{\theta_{2}}\right),
\end{gathered}
$$

where $x_{1}>0, x_{2}>0, a>0, b>0, \theta_{1}>0, \theta_{2}>0$, $\delta \geq 0, \mu \geq 0$, and

$$
K=\left[\theta_{1}^{a} \theta_{2}^{b} \Gamma(a) \Gamma(b) \exp (\delta+\mu)\right]^{-1} .
$$

Now, making the transformation $U=X_{1} /\left(X_{1}+X_{2}\right)$, and $S=X_{1}+X_{2}$ with the Jacobian $J\left(x_{1}, x_{2} \rightarrow\right.$ $u, s)=s$ in (10), the joint density of $U$ and $S$ is obtained as

$$
\begin{gathered}
K u^{a-1}(1-u)^{b-1} \exp \left[-\frac{\{1-(1-\lambda) u\} s}{\theta_{2}}\right] s^{a+b-1} \\
\quad \times{ }_{0} F_{1}\left(a ; \frac{\delta u s}{\theta_{1}}\right){ }_{0} F_{1}\left(b ; \frac{\mu(1-u) s}{\theta_{2}}\right),
\end{gathered}
$$

where $0<u<1$ and $s>0$. Finally, integrating (11) with respect to $s$ by using (8), we obtain the marginal density of $U$.

If the density of $U$ is given by (9), then we will write $U \sim \operatorname{NCGB} 1(a, b ; \lambda ; \delta, \mu)$.

The graphs of the doubly non-central generalized beta type 1 density function for different values of parameters are shown in Figure 1 .

Theorem 3.2. Let $X_{1}$ and $X_{2}$ be independent random variables, $X_{1} \sim \mathrm{Ga}\left(a, \theta_{1}, \delta\right)$ and $X_{2} \sim \mathrm{Ga}\left(b, \theta_{2}, \mu\right)$. Then, $V=X_{1} / X_{2}$ follows a doubly non-central generalized beta type 2 distribution with the the $p d f$

$$
\begin{aligned}
& f_{\mathrm{NCGB} 2}(v ; a, b ; \lambda ; \delta, \mu) \\
& =\frac{\lambda^{a} \exp [-(\delta+\mu)]}{B(a, b)} \frac{v^{a-1}}{(1+\lambda v)^{a+b}} \\
& \quad \times \mathbf{\Psi}_{2}\left[a+b ; a, b ; \frac{\delta \lambda v}{1+\lambda v}, \frac{\mu}{1+\lambda v}\right], v>0,
\end{aligned}
$$

where $\lambda=\theta_{2} / \theta_{1}$.

The above density will be denoted by $V \sim$ $\operatorname{NCGB} 2(a, b ; \lambda ; \delta, \mu)$.

The graphs of the doubly non-central generalized beta type 2 density function for different values of parameters are shown in Figure 2.

For $\delta=\mu=0$, the densities in (9) and (12) slide to generalized beta type 1 and generalized beta type 2 densities, respectively. For $\mu=0$, the densities in (9) and (12) reduce to

$$
\begin{aligned}
& f_{\mathrm{NCGB} 1}(u ; a, b ; \lambda ; \delta, 0) \\
& =\frac{\lambda^{a} \exp (-\delta)}{B(a, b)} \frac{u^{a-1}(1-u)^{b-1}}{[1-(1-\lambda) u]^{a+b}} \\
& \quad \times{ }_{1} F_{1}\left(a+b ; a ; \frac{\delta \lambda u}{1-(1-\lambda) u}\right), 0<u<1
\end{aligned}
$$

and

$$
\begin{aligned}
& f_{\mathrm{NCGB} 2}(v ; a, b ; \lambda ; \delta, 0) \\
& =\frac{\lambda^{a} \exp (-\delta)}{B(a, b)} \frac{v^{a-1}}{(1+\lambda v)^{a+b}} \\
& \quad \times{ }_{1} F_{1}\left(a+b ; a ; \frac{\delta \lambda v}{1+\lambda v}\right), v>0,
\end{aligned}
$$




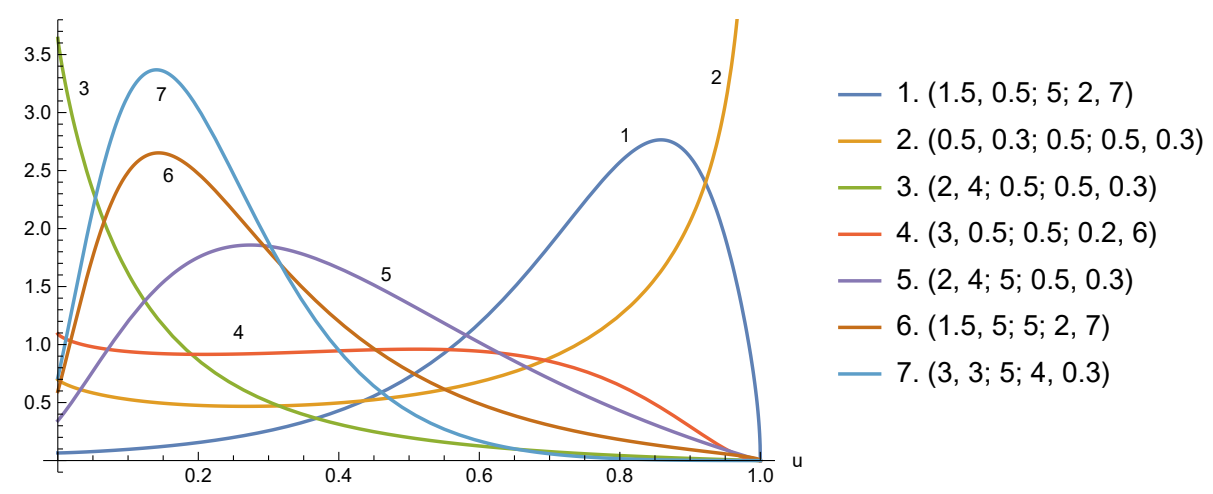

Figure 1: Graph of the density function $f_{\mathrm{NCGB}}(u ; a, b ; \lambda ; \delta, \mu)$ for different values of $(a, b ; \lambda ; \delta, \mu)$
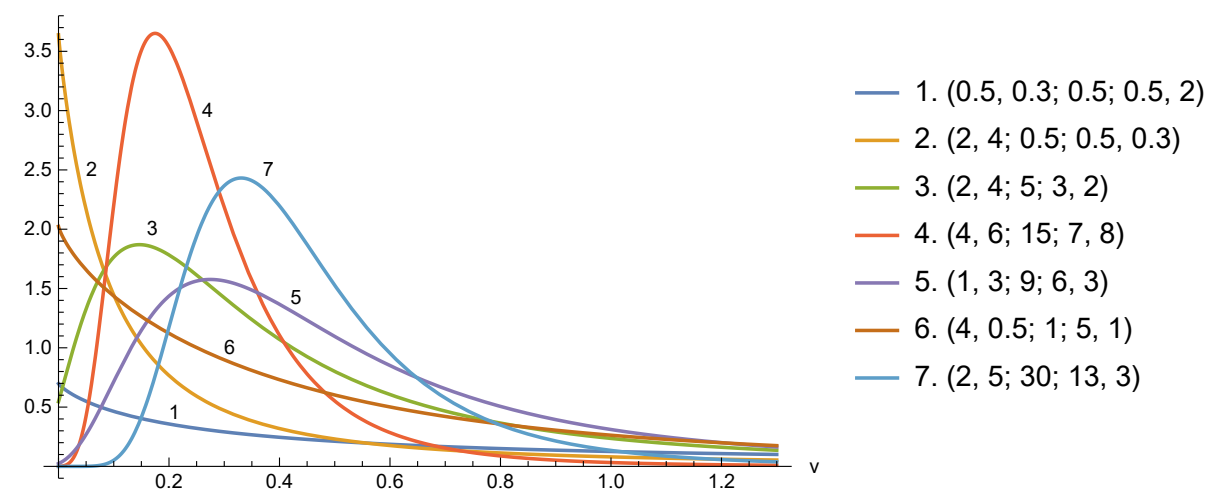

Figure 2: Graphs of the density function $f_{\mathrm{NCGB} 2}(v ; a, b ; \lambda ; \delta, \mu)$ for different values of $(a, b ; \lambda ; \delta, \mu)$

respectively, where ${ }_{1} F_{1}$ is the confluent hypergeometric function.

The distributions defined by densities $(13)$ and (14) will be designated by $\operatorname{NCGB} 1(a, b ; \lambda ; \delta)$ and NCGB2 $(a, b ; \lambda ; \delta)$. In Figure 3 and Figure 4 graphs of NCGB1 and NCGB2 density functions are given for different values of their parameters. Here one can appreciate the wide range of forms that result from the densities (13) and (14).

From the density of the doubly non-central generalized beta type 1 variable, it can be shown that

$$
\begin{aligned}
& \int_{0}^{1} \frac{u^{a-1}(1-u)^{b-1}}{[1-(1-\lambda) u]^{a+b}} \\
& \times \mathbf{\Psi}_{2}\left[a+b ; a, b ; \frac{\delta \lambda u}{1-(1-\lambda) u}, \frac{\mu(1-u)}{1-(1-\lambda) u}\right] \mathrm{d} u \\
& =\exp (\delta+\mu) \lambda^{-a} B(a, b)
\end{aligned}
$$

Using series expansion (7) of the Humbert's confluent hypergeometric function $\boldsymbol{\Psi}_{2}$, it can easily be shown that

$$
\begin{aligned}
& f_{\mathrm{NCGB} 1}(u ; a, b ; \lambda ; \delta, \mu) \\
& =\sum_{r=0}^{\infty} p(r, \delta) f_{\mathrm{NCGB} 1}(u ; a+r, b ; \lambda ; 0, \mu) \\
& =\sum_{s=0}^{\infty} p(s, \mu) f_{\mathrm{NCGB} 1}(u ; a, b+s ; \lambda ; \delta, 0) \\
& =\sum_{r=0}^{\infty} \sum_{s=0}^{\infty} p(r, \delta) p(s, \mu) f_{\mathrm{GB} 1}(u ; a+r, b+s ; \lambda),
\end{aligned}
$$

where $p(x ; \delta)=\exp (-\delta) \delta^{x} / x !, x=0,1,2, \ldots$ is the Poisson density. Thus, from (15), it is clear that the density $f_{\mathrm{NCGB} 1}(u ; a, b ; \lambda ; \delta, \mu)$ is a mixture of central/non-central generalized beta type 1 densities. Similarly, one can show that the non-central beta type 2 density is a mixture of central/non-central generalized beta type 2 densities. That is

$$
\begin{aligned}
& f_{\mathrm{NCGB} 2}(v ; a, b ; \lambda ; \delta, \mu) \\
& =\sum_{r=0}^{\infty} p(r, \delta) f_{\mathrm{NCGB} 2}(v ; a+r, b ; \lambda ; 0, \mu)
\end{aligned}
$$



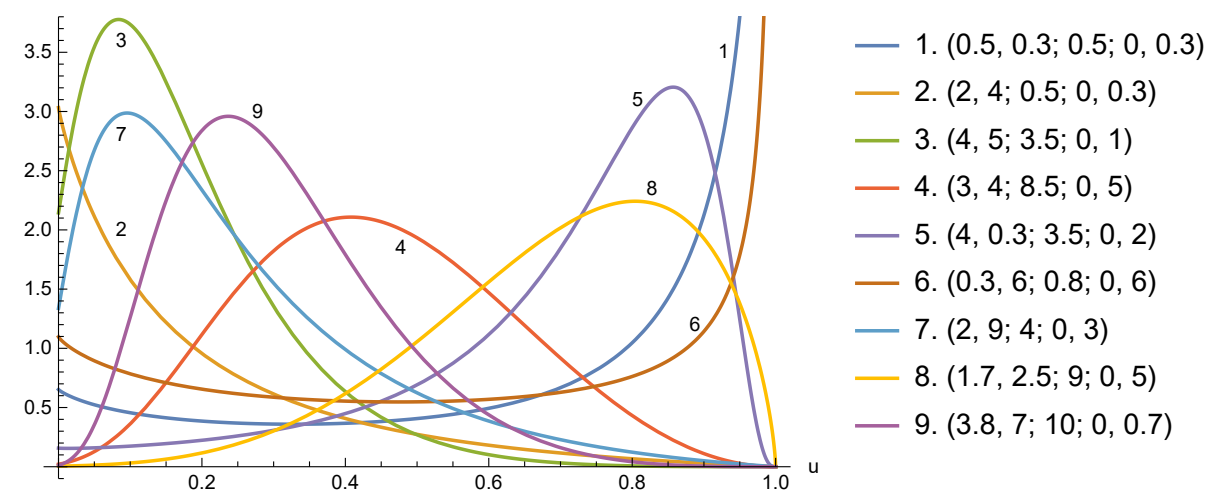

Figure 3: Graphs of the density function $f_{\mathrm{NCGB}}(u ; a, b ; \lambda ; 0, \mu)$ for different values of $(a, b ; \lambda ; 0, \mu)$
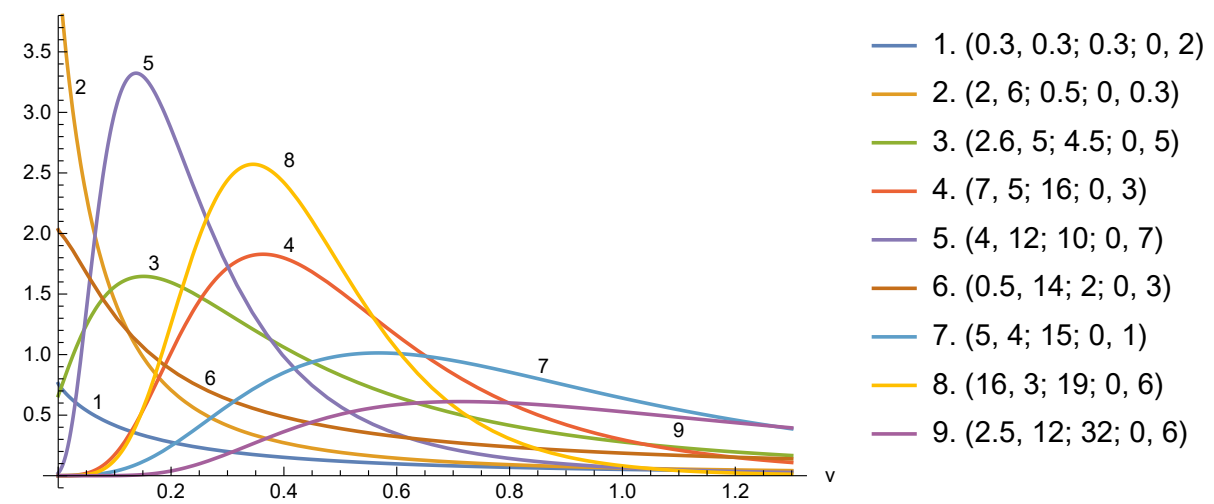

Figure 4: Graphs of density function $f_{\mathrm{NCGB} 2}(v ; a, b ; \lambda ; 0, \mu)$ for different values of $(a, b ; \lambda ; 0, \mu)$

$$
\begin{aligned}
& =\sum_{s=0}^{\infty} p(s, \mu) f_{\mathrm{NCGB} 2}(v ; a, b+s ; \lambda ; \delta, 0) \\
& =\sum_{r=0}^{\infty} \sum_{s=0}^{\infty} p(r, \delta) p(s, \mu) f_{\mathrm{GB} 2}(v ; a+r, b+s ; \lambda) .
\end{aligned}
$$

\section{Expected Values}

Using the series representation of the doubly noncentral generalized beta type 1 density function given in (15), one obtains

$\mathbb{E}\left[\frac{U^{h}(1-U)^{k}}{[1-(1-\lambda) U]^{t}}\right]$

$$
=\sum_{r=0}^{\infty} \sum_{s=0}^{\infty} p(r, \delta) p(s, \mu) \mathbb{E}_{\mathrm{GB} 1}\left[\frac{U^{h}(1-U)^{k}}{[1-(1-\lambda) U]^{t}}\right],
$$

where

$$
\begin{aligned}
& \mathbb{E}_{\mathrm{GB} 1}\left[\frac{U^{h}(1-U)^{k}}{[1-(1-\lambda) U]^{t}}\right] \\
& =\int_{0}^{1} \frac{u^{h}(1-u)^{k}}{[1-(1-\lambda) u]^{t}} f_{\mathrm{GB} 1}(u ; a+r, b+s ; \lambda) \mathrm{d} u
\end{aligned}
$$

and $f_{\mathrm{GB} 1}(u ; a+r, b+s ; \lambda)$ is the generalized beta type 1 density with parameters $a+r, b+s$ and $\lambda$. Now, substituting for $f_{\mathrm{GB} 1}$ in (18) and evaluating the resulting integral by using (6), we have

$$
\begin{aligned}
\mathbb{E}_{\mathrm{GB} 1}\left[\frac{U^{h}(1-U)^{k}}{[1-(1-\lambda) U]^{t}}\right] \\
=\frac{\lambda^{a+r}}{B(a+r, b+s)} \int_{0}^{1} \frac{u^{a+h+r-1}(1-u)^{b+k+s-1}}{[1-(1-\lambda) u]^{a+b+r+s+t}} \mathrm{~d} u \\
=\frac{\lambda^{a+r} B(a+h+r, b+k+s)}{B(a+r, b+s)}{ }_{2} F_{1}(a+h+r, \\
\quad a+b+r+s+t ; a+b+h+k+r+s ; 1-\lambda) .(19)
\end{aligned}
$$

Finally, substituting (19) in (17), the expression for the expected value of $\frac{U^{h}(1-U)^{k}}{[1-(1-\lambda) U]^{t}}$ is given by

$$
\begin{aligned}
& \mathbb{E}\left[\frac{U^{h}(1-U)^{k}}{[1-(1-\lambda) U]^{t}}\right] \\
& =\sum_{r=0}^{\infty} \sum_{s=0}^{\infty} p(r, \delta) p(s, \mu) \frac{\lambda^{a+r} B(a+h+r, b+k+s)}{B(a+r, b+s)} \\
& \quad \times{ }_{2} F_{1}(a+h+r, a+b+r+s+t ; \\
& a+b++k+r+s ; 1-\lambda) .
\end{aligned}
$$


Now, substituting $k=t=0$ in the above expression, the $h$-th moment of $U$ is obtained as

$$
\begin{aligned}
\mathbb{E}\left(U^{h}\right)= & \sum_{r=0}^{\infty} \sum_{s=0}^{\infty} p(r, \delta) p(s, \mu) \\
& \times \frac{\lambda^{a+r} B(a+h+r, b+s)}{B(a+r, b+s)} \\
& \times{ }_{2} F_{1}(a+h+r, a+b+r+s ; \\
& a+b+h+r+s ; 1-\lambda) .
\end{aligned}
$$

Substituting $h=1$ and $h=2$ in (20) and simplifying, we obtains the first and second order moments of the random variable $U$ which has a doubly non-central generalized beta type 1 distribution:

$$
\begin{gathered}
\mathbb{E}(U)=\sum_{r=0}^{\infty} \sum_{s=0}^{\infty} p(r, \delta) p(s, \mu) \frac{\lambda^{a+r}(a+r)}{a+b+r+s} \\
\times{ }_{2} F_{1}(a+1+r, a+b+r+s ; \\
a+b+1+r+s ; 1-\lambda)
\end{gathered}
$$

and

$$
\begin{aligned}
\mathbb{E}\left(U^{2}\right)= & \sum_{r=0}^{\infty} \sum_{s=0}^{\infty} p(r, \delta) p(s, \mu) \\
& \times \frac{\lambda^{a+r}(a+r)(a+r+1)}{(a+b+r+s)(a+b+r+s+1)} \\
& \times{ }_{2} F_{1}(a+2+r, a+b+r+s ; \\
& a+b+2+r+s ; 1-\lambda) .
\end{aligned}
$$

Using the series representation of the doubly noncentral generalized beta type 2 density function given in (16), we get

$$
\begin{aligned}
\mathbb{E}\left[\frac{V^{h}}{(1+\lambda V)^{t}}\right]= & \sum_{r=0}^{\infty} \sum_{s=0}^{\infty} p(r, \delta) p(s, \mu) \\
& \times \mathbb{E}_{\mathrm{GB} 2}\left[\frac{V^{h}}{(1+\lambda V)^{t}}\right]
\end{aligned}
$$

where

$$
\begin{aligned}
& \mathbb{E}_{\mathrm{GB} 2}\left[\frac{V^{h}}{(1+\lambda V)^{t}}\right] \\
& =\int_{0}^{1} \frac{v^{h}}{(1+\lambda v)^{t}} f_{\mathrm{GB} 2}(v ; a+r, b+s ; \lambda) \mathrm{d} v
\end{aligned}
$$

and $f_{\mathrm{GB} 2}(v ; a+r, b+s ; \lambda)$ is the generalized beta type 2 density with parameters $a+r, b+s$ and $\lambda$. Now, substituting $f_{\mathrm{GB} 2}$ in (22) and evaluating the resulting integral, we have

$$
\begin{aligned}
& \mathbb{E}_{\mathrm{GB} 2}\left[\frac{V^{h}}{(1+\lambda V)^{t}}\right] \\
& =\frac{\lambda^{a+r}}{B(a+r, b+s)} \int_{0}^{\infty} \frac{v^{a+h+r-1}}{(1+\lambda v)^{a+b+r+s+t}} \mathrm{~d} u \\
& =\frac{\lambda^{-h} B(a+h+r, b+s+t-h)}{B(a+r, b+s)} .
\end{aligned}
$$

Finally, substituting (23) in (21), the expression for the expected value $\frac{V^{h}}{(1+\lambda V)^{t}}$ is derived as

$$
\begin{aligned}
\mathbb{E}\left[\frac{V^{h}}{(1+\lambda V)^{t}}\right]= & \lambda^{-h} \sum_{r=0}^{\infty} \sum_{s=0}^{\infty} p(r, \delta) p(s, \mu) \\
& \times \frac{B(a+h+r, b+s+t-h)}{B(a+r, b+s)} .
\end{aligned}
$$

Substituting $t=0$ in the above expression, we obtain the $h$-th moment of $V$ as

$$
\begin{aligned}
\mathbb{E}\left(V^{h}\right)= & \lambda^{-h} \sum_{r=0}^{\infty} \sum_{s=0}^{\infty} p(r, \delta) p(s, \mu) \\
& \times \frac{B(a+h+r, b+s-h)}{B(a+r, b+s)} .
\end{aligned}
$$

Further, substituting $h=1$ and $h=2$ in (24) and simplifying, the first and the second order moments of the doubly non-central generalized beta type 2 variable is obtained as

$$
\mathbb{E}(V)=\lambda^{-1} \sum_{r=0}^{\infty} \sum_{s=0}^{\infty} p(r, \delta) p(s, \mu) \frac{a+r}{b+s-1}
$$

and

$$
\begin{aligned}
\mathbb{E}\left(V^{2}\right)= & \lambda^{-2} \sum_{r=0}^{\infty} \sum_{s=0}^{\infty} p(r, \delta) p(s, \mu) \\
& \times \frac{(a+r)(a+r+1)}{(b+s-1)(b+s-2)} .
\end{aligned}
$$

\section{Discussion and Conclusion}

By using the traditional method of transformation of variables, we have obtained probability density functions of doubly non-central generalized beta type 1 and type 2 distributions. These probability density functions have been expressed in terms of the well known Humbert's confluent hypergeometric function $\boldsymbol{\Psi}_{2}$. The doubly non-central generalized beta type 1 and type 2 distributions are generalizations of Libby and Novic's three-parameter beta distributions, beta type 3 distribution, standard non-central beta distributions, and doubly non-central beta distributions. Numerous properties of proposed distributions have also 
been studied. It has also been shown graphically, that doubly non-central generalized beta densities can assume a broad variety of shapes surpassing many existing beta models. Further, infinite mixture representations of doubly non-central generalized beta densities facilitate us to compute cumulative distribution function, moment generating function, and other results in amicable forms.

Further, results such as probability density functions of sum, difference, product and quotient of two independent random variables both having doubly non-central generalized beta type 1 distribution can also be derived. These probability density functions will involve the first hypergeometric function of Appell (see Nagar and Ramirez-Vanegas [10], Nadarajah [20]).

\section{Acknowledgements}

The authors gratefully thank three anonymous referees for their constructive comments and recommendations which definitely helped to improve the readability and quality of the paper.

\section{References:}

[1] Karl Bury, Statistical Distributions in Engineering, Cambridge University Press, Cambridge, 1999.

[2] N. L. Johnson, S. Kotz and N. Balakrishnana, Continuous Univariate Distributions-2, Second Edition, John Wiley \& Sons, New York, 1994.

[3] Z. Kala, Limit states of structures and global sensitivity analysis based on Cramér-von Mises distance, Int. J. Mech., 14, 2020, $107-$ 118.

[4] Yuejun Liu and Huaikou Miao, Bayesian change point estimation based on masked data in exponential distribution parallel system, Int. J. Circuits, Syst. Signal Process, 14, 2020, 28 33.

[5] D. L. Libby and M. R. Novic, Multivariate generalized beta distributions with applications to utility assessment, J. Educ. Statist, 7(4), 1982, 271-294.

[6] J. C. Chen and M. R. Novic, Bayesian analysis for binomial models with generalized beta prior distribution, J. Educ. Statist, 7(2), 1984, no. 2, 163-175.

[7] Liliam Cardeño, D. K. Nagar and Luz Estela Sánchez, Beta type 3 distribution and its multivariate generalization, Tamsui Oxf. J. Math. Sci., 21(1), 2005, 225-241.
[8] A. K. Gupta and D. K. Nagar, Properties of matrix variate beta type 3 distribution, Int. J. Math. Math. Sci., 2009, Art. ID 308518, 18 pp.

[9] Daya K. Nagar and Yeison Arley RamirezVanegas, Non-central beta type 3 distribution, Progress in Applied Mathematics, 4(2), 2012, $29-43$.

[10] Daya K. Nagar and Yeison Arley RamirezVanegas, Distributions of sum, difference, product and quotient of independent noncentral beta type 3 variables, British Journal of Mathematics \& Computer Science, 3(1), 2013, $12-23$.

[11] Daya K. Nagar and Yohana Tabares-Herrera, Entropies and Fisher information matrix for the beta type 3 distribution, Far East J. Math. Sci., 81(1), 2013, 95-102.

[12] T. and Pham-Gia and Q. P. Duong, The generalized beta- and $F$-distributions in statistical modelling, Math. Comput. Modelling, 12(12), 1989, 1613-1625.

[13] A. K. Gupta J. M. Orozco-Castañeda and D. K. Nagar, Non-central bivariate beta distribution, Statist. Papers, 52, 2011, 139-152.

[14] José María Sarabia and Enrique Castillo, Bivariate distributions based on the generalized three-parameter beta distribution, Advances in Distribution Theory, Order Statistics, and Inference, (Balakrishnan N, Sarabia J. M. and Castillo E, editors), Statistics for Industry and Technology. Birkhäuser Boston, Inc., Boston, MA, 2006, 85-110.

[15] Lu Chen and Vijay P. Singh, Generalized beta distribution of the second kind for flood frequency analysis, Entropy, 19(6), 2017, Art. 254.

[16] Emilio Gómez-Déniz and José María Sarabia, A family of generalised beta distributions: properties and applications, Ann. Data Sci., 5, 2018, 401-420. doi/10.1007/s40745-0180143-6

[17] Rafid S. A. Alshkaki, A six parameters beta distribution with application for modeling waiting time of muslim early morning prayer, Ann. Data Sci., Published: 18 May 2020. https://doi.org/10.1007/s40745-020-00282-0

[18] A. K. Gupta and D. K. Nagar, Matrix Variate Distributions, Chapman \& Hall/CRC, Boca Raton, 2000. 
[19] Paulo Renato Alves Firmino, Jair Paulino de Sales, Jucier Gonçalves Júnior, Taciana Araújo da Silva, A non-central beta model to forecast and evaluate pandemics time series, Chaos Solitons Fractals, 140 (11), 2020, Art. 110211.

[20] Saralees Nadarajah, Sums, products, and ratios of non-central beta variables, Comm. Statist. Theory Methods, 34(1), 2005, 89-100.

[21] Y. L. Luke, The Special Functions and Their Approximations, Vol. 1, Academic Press, New York, 1969.

[22] H. M. Srivastava and P. W. Karlsson, Multiple Gaussian Hypergeometric Series, John Wiley \& Sons, New York, 1985.
Creative Commons Attribution License 4.0 (Attribution 4.0 International, CC BY 4.0)

This article is published under the terms of the Creative Commons Attribution License 4.0

https://creativecommons.org/licenses/by/4.0/deed.en_US 